\title{
Review of Clean Development Mechanism and use of Bundled Projects in Small and Medium Scale Enterprises
}

\author{
Mayuri Naik*, Anju Singh, Seema Unnikrishnan, Neelima Naik and Indrayani Nimkar
}

National Institute of Industrial Engineering (NITIE), Mumbai-400087, India

\begin{abstract}
Carbon finance through the Clean Development Mechanism (CDM) offers significant opportunity to a developing country like India for an array of greenhouse gas $(\mathrm{GHG})$ emission reduction projects. However, the transaction cost associated with the development of CDM project is a serious barrier to many small scale CDM (SSC) projects due to which these proponents face many difficulties in attracting international investors. To reduce this transaction cost, individual small projects with similar project context can be bundled together to form a single CDM project. These SSC bundled projects that reduce GHG emissions can claim Certified Emission Reductions (CERs) under the concept of bundling. This paper presents 98 bundled CDM projects registered and issued worldwide till October 2014, out of which India has 29 projects, along with a case study on small scale hydro-electric power generation project. The visited project is a good example of clean technology that helps to reduce stress on conventional energy sources and is an improvement of social and economic life of local people. Energy efficiency, grid connected electricity generation, fossil fuels switching, thermal energy production and methane recovery are some of the methodologies in these types of projects. These methodologies reduce GHG emissions without harming the environment.
\end{abstract}

Keywords: Clean development mechanism; Small scale projects; Bundled; Transaction cost

\section{Introduction}

$\mathrm{CDM}$ is an agreement under Kyoto protocol, which is a multilateral effort taken by United Nation Framework Convention on Climate Change (UNFCCC) to tackle climate change. It is meant to promote changes in the pattern of GHG emission intensive activities in developing countries as discussed in Kyoto, Japan on $11^{\text {th }}$ December 1997 and entered into force on $16^{\text {th }}$ February 2005. 192 countries have ratified the treaty to date. It aims to reduce the GHG emissions by 5.2 $\%$ against the 1990 levels over the first commitment period of five years 2008-2012 [1,2]. Now it has been extended for a second commitment period which is from January 2013 to December 2020. During the second commitment period, parties are committed to reducing their GHG emissions by at least 18 percent below 1990 levels in the coming eight year period from 2013 to 2020 [3]. As a part of CDM, the developed (Annex-I) countries have a target of GHG emission reduction [4]. In order to achieve this target developed countries invest in or finance projects that reduce emissions in developing countries (Non Annex-I) using clean technologies. After implementing these projects in developing countries, for every tone of $\mathrm{CO} 2$ that does not enter into the atmosphere, a developing country earns one CER. These CERs can be further traded in international carbon market. Developed (Annex-I) countries exchange these CERs for money and technology transfer with Developing (Non-Annex-I) countries. Most of the demand for CERs from the CDM comes from the European Union Emissions Trading Scheme (EU-ETS) which is the largest carbon market. $93 \%$ of all the issued CERs come from five developing countries with maximum number of CDM projects. These are China, India, South Korea, Brazil and Mexico [5]. The Conference of Parties (COP), Executive Board (EB), Designated Operational Entity (DOE), Designated National Authority (DNA) are the important regulators in $\mathrm{CDM}$. The COP is the supreme decision making body of the convention. EB supervises the CDM under the authority and guidance of the COP and reviews the regional/sub-regional distribution of the CDM project activities. It also maintains the registry of CDM projects and approves the methodologies for baseline and for monitoring project boundaries.
DOE is an independent auditor accredited by the EB to validate project proposals or verify whether implemented projects have achieved planned GHG emission reductions or CERs. DNA is the organization which has granted the responsibility to authorize and approve CDM projects in respected country. To attain CERs, project developer has to create first a Project Design Document (PDD) which requires the validation by the DOE, preferably by the DNA. Then it is sent to the EB for registration. After the registration, project can run in the developing (host) country. Monitoring is required for measuring CERs. The DNA then verifies and certifies the CERs which are finally issued by the EB. The project developer receives the certificate of CERs at this stage, which further can be sold out in the carbon market. CDM transaction costs are generally fixed. There are substantial diseconomies of scale that make it difficult for small projects to prove their financial viability [6-8]. The impact of such heavy cost warrants careful assessment of risks from potential buyers. Many investors, as well as administrators of carbon funds, would thus consider engaging into small scale CDM ventures only if the estimated potential credits exceed a minimum quota, depending upon their financial assessments. To conquer this, multiple small scale projects of the same type could be bundled together to form a single CDM project. Bundling of small scale projects is better option as it reduces overall costs for the administration of the CDM.

\section{CDM Bundled Projects}

Project activities with a maximum output capacity equivalent of up

*Corresponding author: Mayuri Naik, National Institute of Industrial Engineering (NITIE), Mumbai-400087, India, Tel: 022-28573371; E-mail: mayuri.mrn02@gmail. com

Received September 15, 2015; Accepted September 28, 2015; Published October 05, 2015

Citation: Naik M, Singh A, Unnikrishnan S, Naik N, Nimkar I (2015) Review of Clean Development Mechanism and use of Bundled Projects in Small and Medium Scale Enterprises. Int J Waste Resour 5: 184. doi: 10.4172/2252-5211.1000184

Copyright: ( 2015 Naik MK, et al. This is an open-access article distributed under the terms of the Creative Commons Attribution License, which permits unrestricted use, distribution, and reproduction in any medium, provided the original author and source are credited. 
to 15 megawatts are termed as small scale CDM projects (SSC). SSC projects can be bundled at various stages as $\mathrm{PDD}$ preparation, validation, registration, monitoring, and verification $[9,10]$. The requirements for bundling of SSC projects include: projects should be of the same type, within the same geographical area, at the same stage of development and be bundled by one organization [11]. Further, project activities in a bundle can also be divided in to a series of sub-bundles, provided that each sub-bundle should belong to same type of project activity [12]. Having the same crediting period and composition of bundles shall not change over a period of time. At registration, all project participants have to submit an agreement about their individual project activities to be bundled and their proposed bundling agency should represent all project participants in order to communicate with EB [28]. After implementation of a project, an overall monitoring plan that monitors performance of the constituent project activities further verifies or certifies the emission reductions achieved [13]. A single DOE certifies achieved emission reductions in terms of CERs. So the advantage is that a single validation and certification report for the entire bundle can be obtained, which streamlines these processes for project participants [14]. Bundling of projects could help in achieving a critical mass that would attract financing from lenders. Another advantage in this approach is the sharing of CDM and financing transaction costs among individual projects which could be prohibitive or even create a negative leverage if the transaction is made for a single project [15]. Sometimes it is important to distinguish between a large-sized CDM project and a bundle of small CDM projects because few large sized CDM projects with intension to use the simplified modalities and procedures, and reduce transaction costs may split large projects into two or more small projects. Therefore, the de-bundling of a large project into smaller projects in order to be able to use the simplified procedures is not allowed [16]. As a result of this EB approved a set of criteria to screen out these types of projects to check that a small project truly is a small-scale CDM project by showing that it is not closely related to or integrated with another small-size project. In a bundle, single entity acts on everyone's behalf with lower transaction cost and single monitoring report and allows small scale projects access to carbon market and allows it to benefit from carbon finance. These smaller bundled activities are effectively promoting local sustainable development in impoverished areas of developing countries. This paper provides an insight into these bundled projects which are registered and issued by CDM EB in order to achieve emission reduction in small scale projects. The paper also represents a case study related to small scale CDM bundled project which involves hydroelectric power generation from the water reservoir made available for irrigation. The project was analyzed as it is efficient and environmentally safe technology for power generation.

\section{Results and Discussion}

\section{World}

Figure 1 and Table 1 describe the country wise distribution of the registered CDM bundled projects. A total of $98 \mathrm{CDM}$ bundled projects are registered and issued till July 2014. These projects are categorized on the basis of renewable energy sources used. India is having maximum number of CDM bundled projects (29 numbers), out of which 24 projects deal with wind power, 2 projects are biomass based, 2 projects relate to hydro power and 1project is on energy efficiency. Next to India, China has 26 projects, out of which 23 are power related and 3 are based on other renewable energy sources. 18 and 13 projects in Brazil and Mexico respectively are based on biomass energy. Republic of Korea has

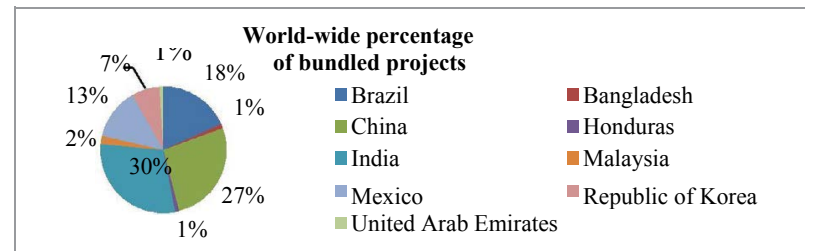

Figure 1: Percentage of Country -wise Bundled projects.

7 projects out of which 3 are based on hydro power, 1 on wind power 1 is related to fuels switching and 2 projects are other renewable energy sources. Malaysia has 2 projects; out of which 1 is based on hydropower and other is biogas based project. Honduras has only 1 project based on biomass energy. Attracting investment to developing countries is a major goal of CDM. United Arab Emirates has 1 project on Energy efficiency. China, India, Brazil, Mexico and Malaysia are the top five countries with maximum number of registered CDM projects and number of issued CERs. These few developing countries are projected to emit more at a faster rate than the industrialized world and the rest of developing countries as these are at the stage of rapid industrialization $[17,18]$. Climate change mitigation projects can reinforce these countries pursuit to a low carbon path of development. Actual emission reduction is the achieved CERs which is the ratio of total CDM credits over a country's actual carbon emissions. It is the expected emission reduction achieved through $\mathrm{CDM}$ projects compared to a country's actual carbon emissions (Table 2). It gives a rough idea of the domestic emission reduction efforts via CDM and how much the CDM projects can contribute to national emission reduction efforts in a given country [19]. Up to July 2014, India has issued maximum number of CERs (1,296,695 metric tons) followed by China issuing $1,175,550$ metric tons of CERs. Brazil and Mexico have issued 601,089 and 317,835 metric tons of CERs respectively. Republic of Korea and Malaysia have so far issued 137,485 and 109,485 metric tons of CERs respectively; United Arab Emirates has issued 2,004 metric tons of CERs. Honduras has so far issued 89,443 metric tons of CERs with maximum CER issuance rate $(104.9 \%)$.

\section{Renewable bundled projects}

The CDM can also interface with renewable energy development to build up the capacity in the developing countries for renewable energy including wind, solar, biomass and hydro. The growth in CER issuance will be driven by capacity additions in the renewable energy sector and by the eligibility of more renewable energy projects to get CERs. Table 3 presents CERs issued under such renewable energy source used in CDM bundled projects across the world and in India respectively. Figure 2 presents renewable energy sources used in worldwide bundled projects. Out of total 98 bundled projects, 33 projects are based on biogas with 940,352 metric tons of CERs issued followed by 29 hydro power projects with maximum issued CERs of 1,393,979 metric tons. 25 projects are based on wind power with 928,386 metric tons. 5 projects are based on other renewable energy sources with 39,999 metric tons of issued CERs. Energy efficiency has 3 projects with 60,494 metric tons of issued CERs. 2 projects are based on biomass with 242,911 metric tons of CERs. Fuel switch have 1 project with issued CERs 6,290 metric tons.

\section{Methodologies}

Methodologies used in CDM projects help to reduce GHG emissions into the atmosphere without harming the environment. The function of 
Citation: Naik M, Singh A, Unnikrishnan S, Naik N, Nimkar I (2015) Review of Clean Development Mechanism and use of Bundled Projects in Small and Medium Scale Enterprises. Int J Waste Resour 5: 184. doi: 10.4172/2252-5211.1000184

Page 3 of 8

\begin{tabular}{|c|c|c|c|c|c|c|}
\hline Sr. no. & Country & No. projects & Type of projects (No. of Projects) & Methodology used & CER Issued & CER Issuance Rate \\
\hline 1 & Brazil & 18 & Biogas (18) & AMS III.D & 601,089 & $53.4 \%$ \\
\hline 2 & Bangladesh & 1 & Energy efficiency (1) & AMS-II.D & 17,403 & $21 \%$ \\
\hline \multirow{2}{*}{3} & \multirow{2}{*}{ China } & \multirow{2}{*}{26} & Hydropower (23) & AMS. I.D & \multirow{2}{*}{$1,175,550$} & \multirow{2}{*}{$68 \%$} \\
\hline & & & Other renewable energy (3) & AMS. I.D & & \\
\hline 4 & Honduras & 1 & Biogas (1) & AMS-I.D. & 170,654 & $117.6 \%$ \\
\hline \multirow{4}{*}{5} & \multirow{4}{*}{ India } & \multirow{4}{*}{29} & Wind power (24) & AMS-I.D. & \multirow{4}{*}{$1,296,695$} & \multirow{4}{*}{$71.3 \%$} \\
\hline & & & Biomass (2) & AMS-I.C. AMS-I.D. & & \\
\hline & & & Hydro (2) & AMS-I.D & & \\
\hline & & & Energy efficiency (1) & AMS-II.D & & \\
\hline \multirow{2}{*}{6} & \multirow{2}{*}{ Malaysia } & \multirow{2}{*}{2} & Hydro (1) & AMS-I.D & \multirow{2}{*}{109,485} & \multirow{2}{*}{$85.60 \%$} \\
\hline & & & Biogas (1) & AMS-III.H. & & \\
\hline 7 & Mexico & 13 & Biogas (13) & AMS-III.D. & 317,835 & $34.70 \%$ \\
\hline \multirow{4}{*}{9} & \multirow{4}{*}{ Republic of Korea } & \multirow{4}{*}{7} & Hydro power(3) & AMS-I.D. & \multirow{4}{*}{137,485} & \multirow{4}{*}{$78.5 \%$} \\
\hline & & & Wind power (1) & AMS-I.D. & & \\
\hline & & & Fuel switch (1) & AMS-III.B. & & \\
\hline & & & Other renewable energy (2) & ACM0002, AMS-I.D. & & \\
\hline \multirow[t]{2}{*}{10} & $\begin{array}{c}\text { United Arab } \\
\text { Emirates }\end{array}$ & 1 & Energy efficiency (1) & AMS-II.D & 2,004 & $20.9 \%$ \\
\hline & & 98 & & & $3,828,200$ & \\
\hline
\end{tabular}

Table 1: Country-wise CDM Bundled Projects.

\begin{tabular}{|c|c|c|c|c|c|c|c|c|c|c|c|}
\hline $\begin{array}{l}\text { Sr. } \\
\text { No. }\end{array}$ & $\begin{array}{c}\text { Name of CDM } \\
\text { Project Activity }\end{array}$ & State & $\begin{array}{c}\text { Other } \\
\text { Parties } \\
\text { Involved }\end{array}$ & $\begin{array}{c}\text { Project Participants } \\
\text { (Authorized by Host } \\
\text { Party) }\end{array}$ & $\begin{array}{c}\text { Project } \\
\text { Participants after } \\
\text { registration } \\
\text { (Authorized by } \\
\text { other Parties } \\
\text { involved) }\end{array}$ & $\begin{array}{l}\text { Type of } \\
\text { Project }\end{array}$ & Methodology & $\begin{array}{l}\text { CER } \\
\text { Issuance } \\
\text { Audits }\end{array}$ & $\begin{array}{l}\text { Total } \\
\text { Issued } \\
\text { CERs }\end{array}$ & $\begin{array}{c}\text { Total } \\
\text { Verified } \\
\text { Days }\end{array}$ & $\begin{array}{l}\text { CER } \\
\text { Issuance } \\
\text { Rate }\end{array}$ \\
\hline 1 & $\begin{array}{l}8.75 \mathrm{MW} \text { Wind } \\
\text { Power Project }\end{array}$ & Gujarat & $\begin{array}{c}\text { Japan; } \\
\text { Switzerland }\end{array}$ & $\begin{array}{c}\text { Rolex Rings Private } \\
\text { Ltd. }\end{array}$ & $\begin{array}{c}\text { Emergent } \\
\text { Ventures India Pvt. } \\
\text { Ltd.; Mitsubishi } \\
\text { Corporation }\end{array}$ & $\begin{array}{l}\text { Wind } \\
\text { power }\end{array}$ & AMS-I.D. & 3 & 44,159 & 1,601 & $64 \%$ \\
\hline 2 & $\begin{array}{c}12 \mathrm{MW} \text { Bundled } \\
\text { Wind Power } \\
\text { Project }\end{array}$ & Tamilnadu & Switzerland & $\begin{array}{c}\text { NEG Micon (I) Private } \\
\text { Ltd. }\end{array}$ & $\begin{array}{l}\text { Vestas Wind } \\
\text { Technology India } \\
\text { Private Limited }\end{array}$ & $\begin{array}{l}\text { Wind } \\
\text { power }\end{array}$ & AMS-I.D. & 2 & 54,732 & 1,027 & $82 \%$ \\
\hline 3 & $\begin{array}{l}\text { Bundled } 15 \mathrm{MW} \\
\text { Wind Power } \\
\text { Project in India }\end{array}$ & $\begin{array}{l}\text { Karnataka, } \\
\text { Tamilnadu } \\
\text { and } \\
\text { Maharashtra }\end{array}$ & $\begin{array}{l}\text { Spain; } \\
\text { Sweden }\end{array}$ & $\begin{array}{l}\text { GNA (HUF), Ferromar } \\
\text { Shipping Private Ltd. }\end{array}$ & $\begin{array}{l}\text { Kingdom of Spain } \\
\text { (withdrawn); } \\
\text { Swedish Energy } \\
\text { Agency (withdrawn) }\end{array}$ & $\begin{array}{l}\text { Wind } \\
\text { power }\end{array}$ & AMS-I.D. & 3 & 127,864 & 1,984 & $67 \%$ \\
\hline 4 & $\begin{array}{l}\text { 3.7 MW Bundled } \\
\text { Wind Power } \\
\text { Project at } \\
\text { Priyadarshini } \\
\text { Polysacks Ltd. }\end{array}$ & Maharashtra & UK & $\begin{array}{l}\text { Priyadarshini } \\
\text { Polysacks Ltd. }\end{array}$ & $\begin{array}{l}\text { The CarbonNeutral } \\
\text { Company Limited }\end{array}$ & $\begin{array}{l}\text { Wind } \\
\text { power }\end{array}$ & AMS-I.D. & 6 & 28,404 & 2,193 & $61 \%$ \\
\hline 5 & $\begin{array}{c}\text { 13.4 MW bundled } \\
\text { wind power } \\
\text { project }\end{array}$ & Karnataka & $\begin{array}{c}\text { Norway; } \\
\text { Switzerland }\end{array}$ & $\begin{array}{c}\text { NEG Micon (I) Private } \\
\text { Ltd. }\end{array}$ & $\begin{array}{l}\text { The Norwegian } \\
\text { Ministry of Finance; } \\
\text { Vestas Wind } \\
\text { Technology India } \\
\text { Private Limited }\end{array}$ & $\begin{array}{l}\text { Wind } \\
\text { power }\end{array}$ & AMS-I.D. & 3 & 125,015 & 2,033 & $102 \%$ \\
\hline 6 & $\begin{array}{l}11.2 \mathrm{MW} \text { Wind } \\
\text { Power project }\end{array}$ & Tamilnadu & Switzerland & $\begin{array}{l}\text { Amarjothi Spinning } \\
\text { mills Ltd. }\end{array}$ & Vitol S.A. & $\begin{array}{l}\text { Wind } \\
\text { power }\end{array}$ & AMS-I.D. & 2 & 158,386 & 2,555 & $92 \%$ \\
\hline 7 & $\begin{array}{c}7.85 \mathrm{MW} \\
\text { Bundled Wind } \\
\text { Power Project in } \\
\text { Southern India }\end{array}$ & $\begin{array}{l}\text { karnataka } \\
\text { and } \\
\text { Tamilnadu }\end{array}$ & Switzerland & Vandana Ispat Ltd. & $\begin{array}{l}\text { Bunge Emissions } \\
\text { Fund Limited }\end{array}$ & $\begin{array}{l}\text { Wind } \\
\text { power }\end{array}$ & AMS-I.D. & 1 & 16,494 & 385 & $66 \%$ \\
\hline 8 & $\begin{array}{c}\text { Bundled } 3.0 \mathrm{MW} \\
\text { Wind Energy } \\
\text { Project }\end{array}$ & Tamilnadu & Switzerland & $\begin{array}{c}\text { M/s Bhagyodaya } \\
\text { Agencies }\end{array}$ & $\begin{array}{l}\text { Emergent Ventures } \\
\text { India (Pvt) Ltd. }\end{array}$ & $\begin{array}{l}\text { Wind } \\
\text { power }\end{array}$ & AMS-I.D. & 1 & 10,373 & 750 & $68 \%$ \\
\hline 9 & $\begin{array}{l}\text { 8.75MW Bundle } \\
\text { Wind Power } \\
\text { Project }\end{array}$ & Maharashtra & -- & $\begin{array}{c}\text { M/s Shahi Export } \\
\text { Pvt. Ltd }\end{array}$ & -- & $\begin{array}{l}\text { Wind } \\
\text { power }\end{array}$ & AMS-I.D. & 1 & 32,977 & 976 & $59 \%$ \\
\hline 10 & $\begin{array}{l}4 \text { MW Bundled } \\
\text { Grid Connected } \\
\text { Wind Power } \\
\text { Project }\end{array}$ & Tamilnadu & Spain & $\begin{array}{l}\text { Pushpit Steels Private } \\
\text { Ltd. ; V. S. Steel and } \\
\text { Power Private Ltd. ; } \\
\text { Garg Iron \& Energy } \\
\text { Private Ltd. }\end{array}$ & $\begin{array}{c}\text { Zero Emissions } \\
\text { Technologies S.A. }\end{array}$ & $\begin{array}{l}\text { Wind } \\
\text { power }\end{array}$ & AMS-I.D. & 2 & 19,180 & 1,102 & $76 \%$ \\
\hline
\end{tabular}


Citation: Naik M, Singh A, Unnikrishnan S, Naik N, Nimkar I (2015) Review of Clean Development Mechanism and use of Bundled Projects in Small and Medium Scale Enterprises. Int J Waste Resour 5: 184. doi: 10.4172/2252-5211.1000184

Page 4 of 8

\begin{tabular}{|c|c|c|c|c|c|c|c|c|c|c|c|}
\hline 11 & $\begin{array}{l}\text { 7.2 MW Wind } \\
\text { Project at } \\
\text { Chitradurga }\end{array}$ & Karnataka & Japan & $\begin{array}{l}\text { Mysore Mercantile } \\
\text { Company Ltd. }\end{array}$ & \begin{tabular}{|c|} 
Mitsubishi UFJ \\
Morgan Stanley \\
Securities Co., Ltd.
\end{tabular} & $\begin{array}{l}\text { Wind } \\
\text { power }\end{array}$ & AMS-I.D. & 5 & 62,478 & 1,796 & $88 \%$ \\
\hline 12 & $\begin{array}{l}\text { 8.5 MW Wind } \\
\text { Energy Project by } \\
\text { KS Oils Limited, } \\
\text { India }\end{array}$ & $\begin{array}{l}\text { Madhya } \\
\text { Pradesh and } \\
\text { Gujarat }\end{array}$ & Switzerland & K.S. Oils Ltd. & $\begin{array}{l}\text { Emergent Ventures } \\
\text { India Pvt. Ltd. }\end{array}$ & $\begin{array}{l}\text { Wind } \\
\text { power }\end{array}$ & AMS-I.D. & 3 & 35,827 & 962 & $81 \%$ \\
\hline 13 & $\begin{array}{l}\text { 2.5 MW Bundled } \\
\text { Wind Power } \\
\text { Project }\end{array}$ & Maharashtra & -- & $\begin{array}{c}\text { Raj Infrastructure } \\
\text { Development, (India) } \\
\text { Pvt. Ltd. }\end{array}$ & -- & $\begin{array}{l}\text { Wind } \\
\text { power }\end{array}$ & AMS-I.D. & 2 & 6,319 & 750 & $77 \%$ \\
\hline 14 & $\begin{array}{l}\text { Bundled Wind } \\
\text { Power Project }\end{array}$ & Tamil Nadu & Switzerland & $\begin{array}{c}\text { Jocil Ltd } \\
\text { The Andhra Sugars } \\
\text { Ltd }\end{array}$ & $\begin{array}{l}\text { Bunge Emissions } \\
\text { Fund Limited }\end{array}$ & $\begin{array}{l}\text { Wind } \\
\text { power }\end{array}$ & AMS-I.D. & 2 & 57,479 & 765 & $80 \%$ \\
\hline 15 & $\begin{array}{l}\text { 5.5 MW Bundled } \\
\text { Wind Power } \\
\text { Project by WMI } \\
\text { Cranes Ltd. }\end{array}$ & $\begin{array}{l}\text { Maharashtra, } \\
\text { Gujarat and } \\
\text { Tamil Nadu }\end{array}$ & Switzerland & WMI Cranes Ltd. & $\begin{array}{c}\text { RWE Supply \& } \\
\text { Trading Switzerland } \\
\text { S.A. }\end{array}$ & $\begin{array}{l}\text { Wind } \\
\text { power }\end{array}$ & AMS-I.D. & 1 & 7,991 & 565 & $45 \%$ \\
\hline 16 & $\begin{array}{l}7.5 \mathrm{MW} \text { bundled } \\
\text { small-scale wind } \\
\text { projec }\end{array}$ & Maharashtra & $\begin{array}{l}\text { Spain; } \\
\text { Sweden }\end{array}$ & $\begin{array}{l}\text { Modelama Exports } \\
\text { Ltd. }\end{array}$ & $\begin{array}{l}\text { Kingdom of Spain } \\
\text { (withdrawn); } \\
\text { Swedish Energy } \\
\text { Agency (withdrawn) }\end{array}$ & $\begin{array}{l}\text { Wind } \\
\text { power }\end{array}$ & AMS-I.D. & 3 & 26,900 & 981 & $76 \%$ \\
\hline 17 & $\begin{array}{c}9.75 \mathrm{MW} \\
\text { Bundled wind } \\
\text { power project }\end{array}$ & Kerala & -- & $\begin{array}{l}\text { Zenith Energy } \\
\text { Services }(P) \text { Ltd. }\end{array}$ & -- & $\begin{array}{l}\text { Wind } \\
\text { power }\end{array}$ & AMS-I.D. & 1 & 19,160 & 370 & $89 \%$ \\
\hline 18 & $\begin{array}{l}\text { Bundled Wind } \\
\text { Power Project } \\
\text { by M/s. D. J. } \\
\text { Malpani }\end{array}$ & $\begin{array}{l}\text { Karnataka } \\
\text { and Gujarat }\end{array}$ & -- & M/s. D. J. Malpani & -- & $\begin{array}{l}\text { Wind } \\
\text { power }\end{array}$ & AMS-I.D. & 1 & 22,285 & 631 & $114 \%$ \\
\hline 19 & $\begin{array}{l}\text { Wind Power } \\
\text { Generation } \\
\text { Project activity } \\
\text { by Interocean } \\
\text { Shipping India } \\
\text { Private Limited }\end{array}$ & $\begin{array}{c}\text { Maharashtra, } \\
\text { Tamilnadu } \\
\text { and Rajsthan }\end{array}$ & Switzerland & $\begin{array}{l}\text { Interocean Shipping } \\
\text { India Private Ltd. }\end{array}$ & EGL AG & $\begin{array}{l}\text { Wind } \\
\text { power }\end{array}$ & AMS-I.D. & 1 & 10,292 & 370 & $46 \%$ \\
\hline 20 & $\begin{array}{l}\text { Cleaner } \\
\text { Technology } \\
\text { in Electricity } \\
\text { Production }\end{array}$ & Tamil Nadu & -- & Sheela Clinic & -- & $\begin{array}{l}\text { Wind } \\
\text { power }\end{array}$ & AMS-I.D. & 1 & 6,403 & 322 & $86 \%$ \\
\hline 21 & $\begin{array}{l}\text { Bundled Wind } \\
\text { Power Project }\end{array}$ & Gujarat & -- & $\begin{array}{l}\text { Vish Wind } \\
\text { Infrastructure LLP ; } \\
\text { J. N. Investment \& } \\
\text { Trading Co. Private } \\
\text { Ltd. }\end{array}$ & -- & $\begin{array}{l}\text { Wind } \\
\text { power }\end{array}$ & AMS-I.D. & 1 & 17,732 & 396 & $54 \%$ \\
\hline 22 & $\begin{array}{c}4.75 \mathrm{MW} \\
\text { Bundled Wind } \\
\text { Power Project } \\
\text { by Associated } \\
\text { Stone Industries } \\
\text { (Kotah) Ltd }\end{array}$ & $\begin{array}{l}\text { Tamil Nadu, } \\
\text { Karnataka } \\
\text { and } \\
\text { Maharashtra }\end{array}$ & -- & $\begin{array}{c}\text { Associated Stone } \\
\text { Industries (Kotah) Ltd. }\end{array}$ & -- & $\begin{array}{l}\text { Wind } \\
\text { power }\end{array}$ & AMS-I.D. & 1 & 4,010 & 366 & $34 \%$ \\
\hline 23 & $\begin{array}{c}\text { 9.9 MW Bundled } \\
\text { Wind Power } \\
\text { Project }\end{array}$ & Maharashtra & -- & REI Agro Ltd. & -- & $\begin{array}{l}\text { Wind } \\
\text { power }\end{array}$ & AMS-I.D. & 1 & 14,017 & 385 & $58 \%$ \\
\hline 24 & \begin{tabular}{|c|} 
Bundled 9.00 \\
MW Wind Power \\
Generation \\
Project by \\
Gangadhar \\
Narsingdas \\
Agrawal Group
\end{tabular} & $\begin{array}{c}\text { Maharashtra } \\
\text { and } \\
\text { Karnataka }\end{array}$ & -- & $\begin{array}{c}\text { Gangadhar } \\
\text { Narsingdas Agrawal } \\
\text { (HUF) ; Ferromar } \\
\text { Shipping Private Ltd. } \\
\text { (FSPL) }\end{array}$ & -- & $\begin{array}{l}\text { Wind } \\
\text { power }\end{array}$ & AMS-I.D. & 1 & 17,615 & 355 & $99 \%$ \\
\hline 25 & $\begin{array}{c}2 \times 5 \mathrm{MW} \\
\text { Upper khauli \& } \\
\text { Drinidhar small } \\
\text { hydroelectric } \\
\text { project for a grid } \\
\text { system }\end{array}$ & $\begin{array}{l}\text { Himachal } \\
\text { Pradesh }\end{array}$ & -- & $\begin{array}{l}\text { Vamshi Industrial } \\
\text { Power Ltd. }\end{array}$ & -- & $\begin{array}{l}\text { Hydro } \\
\text { power }\end{array}$ & AMS-I.D. & 1 & 31,643 & 731 & $43 \%$ \\
\hline 26 & $\begin{array}{l}10 \mathrm{MW} \\
\text { Renewable } \\
\text { Energy Project } \\
\text { for a Grid }\end{array}$ & $\begin{array}{l}\text { Himachal } \\
\text { Pradesh }\end{array}$ & -- & $\begin{array}{l}\text { AT Hydro (P) Ltd. ; } \\
\text { Cimaron Power Ltd. }\end{array}$ & -- & $\begin{array}{l}\text { Hydro } \\
\text { power }\end{array}$ & AMS-I.D. & 1 & 54,962 & 730 & $70 \%$ \\
\hline
\end{tabular}




\begin{tabular}{|c|c|c|c|c|c|c|c|c|c|c|c|}
\hline 27 & $\begin{array}{l}7.5 \mathrm{MW} \text { biomass } \\
\text { plants using } \\
\text { agricultural waste } \\
\text { Limited }\end{array}$ & Tamilnadu & Germany & $\begin{array}{l}\text { Shriram City Union } \\
\text { Finance Ltd. Shriram } \\
\text { Transport Finance } \\
\text { Company Ltd. } \\
\text { (Shriram nvestments } \\
\text { Ltd. }\end{array}$ & KfW ('withdrawn) & Biomass & AMS-I.D. & 2 & 190,303 & 1,362 & $63 \%$ \\
\hline 28 & $\begin{array}{c}\text { Renewable } \\
\text { biomass based } \\
\text { thermal energy } \\
\text { generation at } \\
\text { Mahalaxmi Group } \\
\text { of Companies }\end{array}$ & Gujarat & -- & $\begin{array}{c}\text { Mahalaxmi Fabric Mills } \\
\text { Private Ltd. (MFM) } \\
\text { Mahalaxmi Rubtech } \\
\text { Ltd. (MRT) }\end{array}$ & & Biomass & AMS-I.C. & 2 & 52,608 & 1,430 & $39 \%$ \\
\hline 29 & $\begin{array}{l}\text { Fuel efficiency } \\
\text { improvement in } \\
\text { glass melting }\end{array}$ & $\begin{array}{l}\text { West Bengal, } \\
\text { Union } \\
\text { Territory of } \\
\text { Pondicherry } \\
\text { and Haryana }\end{array}$ & Switzerland & $\begin{array}{l}\text { Hindusthan National } \\
\text { Glass \& Industries Ltd. }\end{array}$ & $\begin{array}{l}\text { Mercuria Energy } \\
\text { Trading SA }\end{array}$ & $\begin{array}{l}\text { Energy } \\
\text { efficiency }\end{array}$ & AMS-II.D. & 1 & 41,087 & 1,096 & $90 \%$ \\
\hline & & 29 & & & 17 & 29 & & 55 & $1,296,695$ & 28,969 & $71.3 \%$ \\
\hline
\end{tabular}

Table 2: The List of Bundled CDM Projects in India

\begin{tabular}{|c|c|c|c|c|}
\hline \multirow{2}{*}{ Renewable Energy Source } & \multicolumn{2}{|c|}{ No. of Projects } & \multicolumn{2}{|c|}{ No. of CERs issued } \\
\hline & World & India & World & India \\
\hline Wind power & 25 & 24 & 928386 & $9,26,092$ \\
\hline Biomass & 2 & 2 & $2,42,911$ & $2,42,911$ \\
\hline Biogas & 33 & 0 & $11,56,141$ & 0 \\
\hline Hydro power & 29 & 2 & $13,93,979$ & 86,605 \\
\hline Fuel Switch & 1 & 0 & 6,290 & 0 \\
\hline Energy efficiency & 3 & 1 & 60,494 & 41,087 \\
\hline Other Renewable & 5 & 0 & 39,999 & 0 \\
\hline
\end{tabular}

Table 3: CERs issued with respect to Renewable energy sources in World (including India).

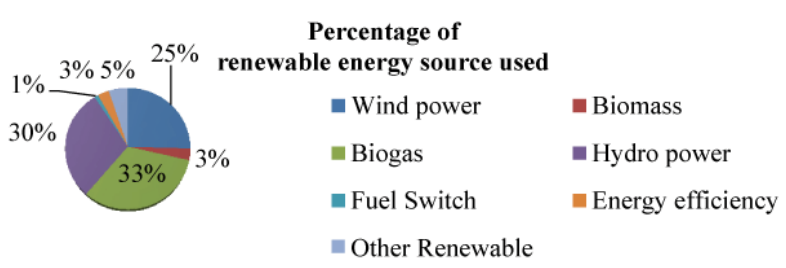

Figure 2: Worldwide renewable energy source used.

these methodologies is easy to grasp, but the methodologies themselves can be quite complex. These are necessarily diverse in their composition and application in order to accommodate the wide range of activities and areas covered by the CDM [20]. The CDM Executive Board approves all baseline-monitoring methodologies. It takes around 200 days between the submission of a new methodology and final decision on rejection; about 300 days are required to get the final approval decision [21]. Energy efficiency, renewable energy, landfill gas recovery, biomass and methane recovery [22] are the types of methodologies mostly used in simplified small scale projects which helps reduce the transaction cost of the project $[23,24]$. Table 4 and Figure 3 provides the list of all the methodologies that are used in all 98 bundled projects. Only 7 different methodologies were used in 98 bundled projects. All are approved small scale methodologies. Methodology "Grid connected renewable electricity generation" (AMS-I.D) contributes to 58 projects followed by "Methane recovery in animal manure management systems" (AMSIII.D) used in 31 projects. Methodologies "Energy efficiency and fuel switching measures for industrial facilities" (AMS-II.D.) contributes to 3 projects followed by "Thermal energy production with or without electricity" (AMS-I.C.) in 2 projects, "Grid-connected electricity generation from renewable sources" (ACM0002) were executed in 2

\begin{tabular}{|l|l|l|l|}
\hline Sr. No. & Methodology & No. of Project & Name of the Methodology \\
\hline 1 & AMS-I.D & 58 & $\begin{array}{l}\text { Grid connected renewable electricity } \\
\text { generation }\end{array}$ \\
\hline 2 & AMS-II.D. & 3 & $\begin{array}{l}\text { Energy efficiency and fuel switching } \\
\text { measures for industrial facilities }\end{array}$ \\
\hline 3 & AMS-III.D. & 31 & $\begin{array}{l}\text { Methane recovery in animal manure } \\
\text { management systems }\end{array}$ \\
\hline 4 & AMS-I.C. & 2 & $\begin{array}{l}\text { Thermal energy production with or } \\
\text { without electricity }\end{array}$ \\
\hline 5 & AMS-III.H. & 1 & $\begin{array}{l}\text { Methane recovery in wastewater } \\
\text { treatment }\end{array}$ \\
\hline 6 & AMS-III.B. & 1 & Switching fossil fuels \\
\hline 7 & ACM0002 & 2 & $\begin{array}{l}\text { Grid-connected electricity generation } \\
\text { from renewable sources }\end{array}$ \\
\hline
\end{tabular}

Table 4: Methodologies used in worldwide Bundled CDM Projects.

projects. "Methane recovery in wastewater treatment" (AMS-III.H.) and "Switching fossil fuels" (AMS-III.B.) were implemented in 1 project each. Figure 4 shows methodology "Grid connected renewable electricity generation" (AMS.I.D) was used in maximum number of projects. The methodology involves construction and the operation of a power plant that uses renewable energy sources and supplies electricity to the grid or retrofit, replacement or capacity addition of an existing power plant that uses renewable energy sources and supplies electricity to the grid (CDM Rule Book, 2013). It is an approved methodology for small-scale grid-connected renewable energy projects that have less than $15 \mathrm{MW}$ threshold capacity [25]. This methodology is applicable to all type of projects that use renewable energy sources like wind, hydro, solar and biomass. Energy generated through CDM projects is supplied to the national grid which increases energy availability. This energy can be supplied to areas having shortage of electricity and help in reducing load on national and local grids as well as in community welfare. 


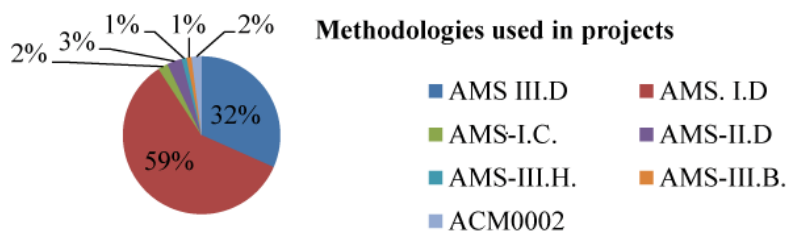

Figure 3: Worldwide methodologies used.

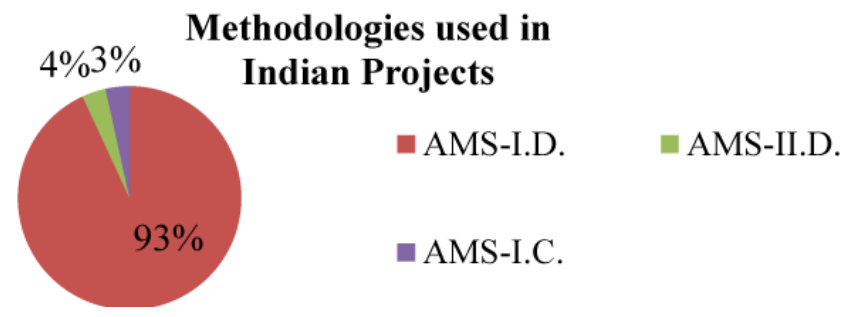

Figure 4: Methodologies used in India.

\section{India}

India has a long dependency on traditional energy sources such as firewood, agricultural waste, animal dung etc., which are still continuing to meet the energy necessities especially in rural India. These traditional fuels are getting replaced by fossil fuels such as coal, petroleum and natural gas which are major cause of climatic change and air pollution. Thus, the focus of energy planners has shifted towards renewable resources like wind, solar, biogas for energy generation [26]. Table 2 presents the list of CDM bundled projects in India that use renewable resources and furnish the details of bundled project activities, such as project participants, use of renewable energy sources in the projects, methodologies used in these projects and estimated emission reduction per annum with respective number of issued CERs in the year 2014 for its total verified days and CERs issuance rate. India has 29 registered bundled projects till July 2014 having 1,296,695 metric tons of CERs issued with $71.3 \%$ issuance rate. In India bundled projects have been implemented in wind power, biomass based hydro power and energy efficiency sectors with the help of different methodologies. Figure 5 presents types of renewable energy source used in Indian bundled projects. Out of 29 bundled projects, 24 are based on wind power with 881,161 metric tons of issued CERs. 2 hydro power and biomass based project each with 54,962 and 242,911 metric tons of CERs respectively. 1 project is on energy efficiency with 41,057 metric tons of issued CERs. Wind power based projects have used AMS.I.D methodology with 926,092 CER issued with 73.5\% CER issuance rate, biomass based projects implemented AMS.I.D and AMS.I.C methodologies with 242,911 CER issued with 50.8\% CER issuance rate and energy efficiency used AMS.II.D methodology with 41,087 issued CER with $90 \%$ of CER issuance rate and hydropower based projects implemented AMS.I.D methodology with 86,605 CER issued with $56.5 \%$ of CERs issuance rate. Figure 6 represents number of bundled projects in different states of India. Karnataka, Tamil Nadu, Maharashtra, Gujarat, Madhya Pradesh, Kerala, Rajasthan, Himachal Pradesh, West Bengal, Haryana, Pondicherry are the states where bundled projects are being implemented. Out of 24 wind power

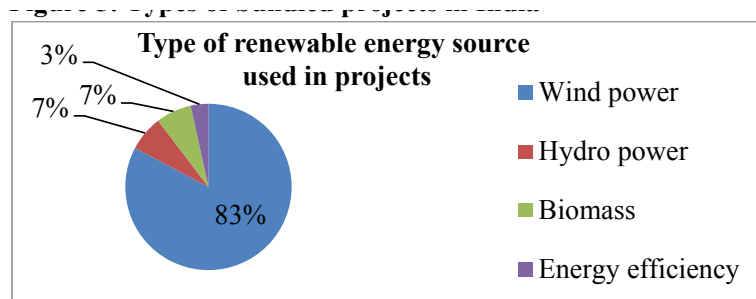

Figure 5: Types of bundled projects in India.
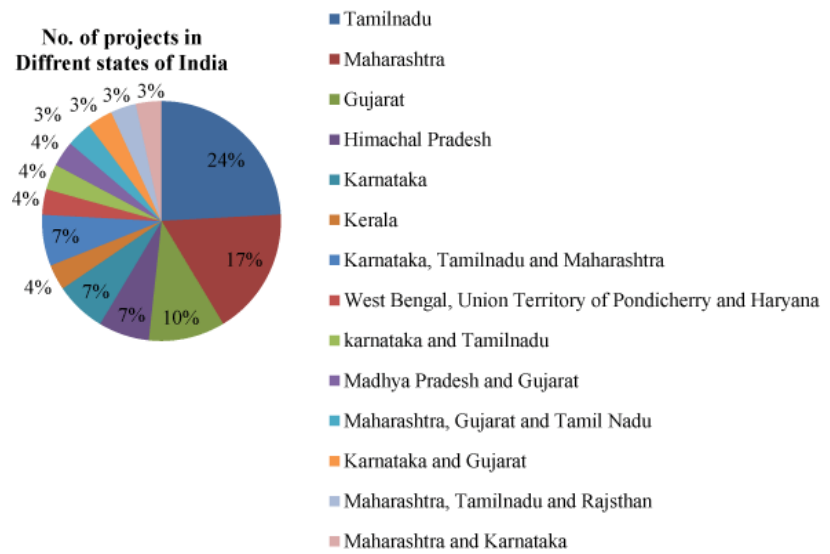

Figure 6: Bundled projects in different states of India.

projects in India, maximum projects are carried out in Tamil Nadu (6) and Maharashtra (5) followed by Karnataka and Gujarat with 2 projects each and Kerala with only 1 project. Tamil Nadu and Gujarat each have 1 biomass based CDM bundled project. Himachal Pradesh has 1 hydropower based project. Within 29 projects in India, 5 projects have achieved below 50\% CERs. 10 projects have achieved CERs between $50 \%-70 \%$. Other 10 projects have achieved CERs within $70-90 \%$. Only 4 projects have achieved CERs more than $90 \%$. In India maximum projects have used wind energy for power generation. These projects use wind energy for power generation thereby reducing usage of fossil fuel which leads to reduction of GHGs emission into the atmosphere. Wind turbine generators deployed in these projects ensure efficient and safe operation of the project activity. Thus the mechanism establishes technical expertise in countries with little prior experience, helping to incentivize increasing amounts of foreign investment (Hedger and Stokes, Undated). Grid-interactive power generated from renewable sources in the year 2013 was $4125 \mathrm{MW}$, out of which wind power generation was maximum $2500 \mathrm{MW}, 350 \mathrm{MW}$ power is obtained from Small Hydro Power and Bagasse Cogeneration each, $105 \mathrm{MW}$ power is from Biomass \& Gasification, Waste to power generation was $20 \mathrm{MW}$ and power generated from solar energy was $800 \mathrm{MW}$ [27].

\section{Case Study}

The visited project is the Dhom (Balkawadi) water reservoir which has capacity of 115.53 million cubic meters. It is built across flow of Krishna River in Balakwadi village, Wai Taluka, Satara district, Maharashtra state, India. The project is a 'bundled project activity' with a total installed capacity of $6.5 \mathrm{MW}$ electricity generations. Projects are typical 'dam-toe' irrigation based river hydro projects with the installation of power house at the foot of an existing dam. The projects utilize irrigation discharges from the storage across river and surplus discharges through the spillway to generate electricity. The electricity 


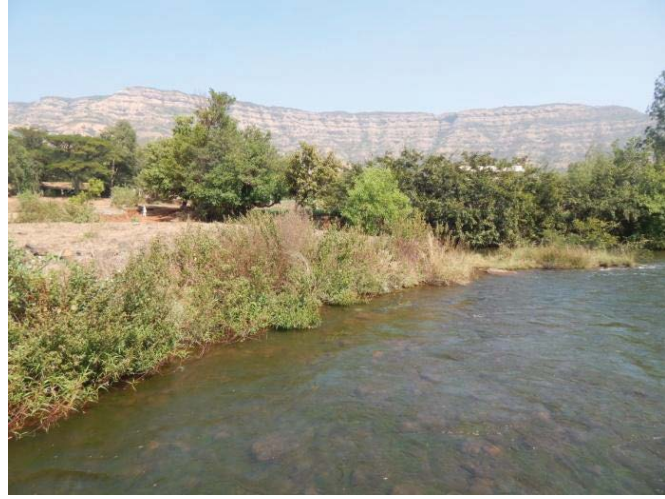

Figure 7: Total Catchment Area

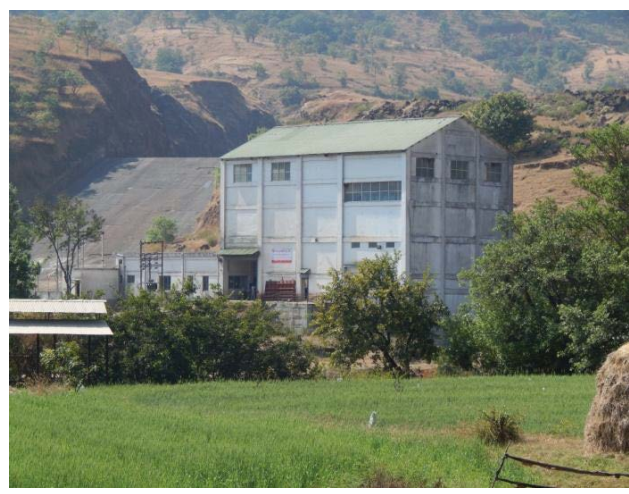

Figure 8: Powerhouse

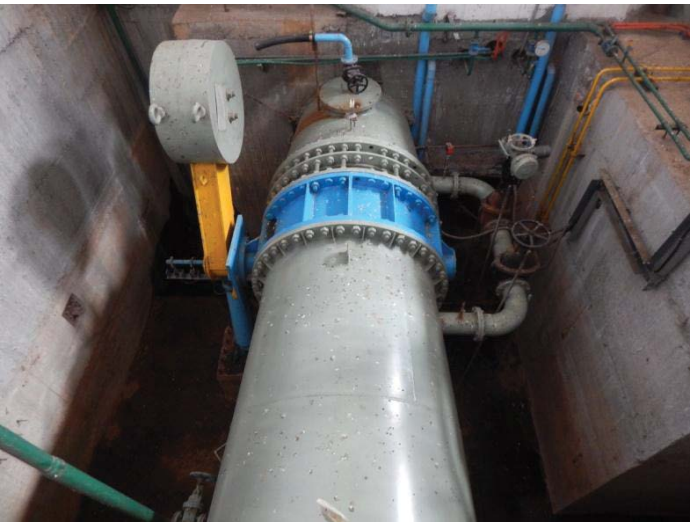

Figure 9: Penstock

is mainly supplied to co-operatives, industries, educational institutions and other sectors. The total catchment area (Figure 7) is around $42.77 \mathrm{~km}^{2}$. The length of the dam is $1.173 \mathrm{~km}$. Dam serves the purpose of storing water for irrigation. Released water is stored downstream. The irrigation is from the submergence of Dhom backwaters. The water stored in the dam is released into the reservoir through tunnel. Project envisages the construction of a small powerhouse of (Figure 8) capacity $4 \mathrm{MW}$ to be operated by the water released from the reservoir made available for irrigation. The annual net generation of the project is estimated at $9.37 \mathrm{MW}$ [28]. A pipe between the surge tank and prime mover and a butterfly wall, which is used to regulate the flow in a pipe, is called a penstock (Figure 9). Penstock is made of steel through reinforced concrete and usually equipped with the head gates at the inlet which can be closed during the repair of the penstocks. A sufficient water head should be provided above the penstock entrance in the fore bay or surge tank to avoid the formation of vortices. The butterfly valve consists of a disk built onto a rod, which when turned to the closed position completely stops the flow. When the butterfly valve is in the open position, the flow is nearly unrestricted. A turbine device with blades at one end and electromagnets at the other end, generates electricity as the blades moves. It consists of different parts viz. generator, shaft, and guard wheel. The online monitoring of electricity is done on continuously. The meters are capable of measuring the electricity parameters on real time basis. Data is archived in electronic form for two years after the end of the crediting period or of the last issuance of CERs. The generated electricity is connected to grid which supplies electricity to an identified consumer facility. On-site small scale renewable energy generation projects can produce significant clean energy, environmental, and economic benefits for a local government and the community. Hydropower is one of the highest renewable energy resources used after wind. Also, it contributes to grid stability by providing flexibility, as spinning turbines can be ramped up more rapidly than any other generation source [29]. Cited small scale hydro-power project is good example clean power generation. The project leads to conservation of resources as the power facility that would have come up in the absence of the conventional energy sources. It has the potential to shape the social and economic life of the local people by creating jobs on a regular and permanent basis and additional investment consistent with the needs of the local community. It is expected to accelerate the commercialization of grid connected renewable energy technologies such as small hydro in the market and thus, provide an alternative to the high-growth, coal-dominated business as-usual scenario.

\section{Recommendation Suggested as per the Study}

India is still depending on traditional energy sources to fulfill their energy needs. Whereas traditional energy sources are non-renewable and limited in supply also causes climatic change and air pollution. Thus, to minimize these effects energy-use pattern have changed towards renewable energy resources like wind, solar, hydro, biogas. Rapid deployment of renewable energy resources are resulting in significant energy security, climate change mitigation, and economic benefits. CDM can interface with renewable energy development projects to build up the capacity for renewable energy in developing countries. But small scale energy generation projects, which are typically found in less developed countries, face proportionally higher transaction costs. Therefore, approach in the concept of bundling of small scale CDM projects is to share CDM financing transaction cost between individual projects. Bundling of projects will facilitate the scaling-up of carbon finance business, while minimizing the transaction costs per unit emission reductions. Many small bundled and large scale renewable energy projects are serving as climate change mitigation projects. Also, these projects keep money circulating within the local economy and would reduce the need to spend money on importing coal and natural gas from other places. Thus, they projects have high potential to contribute local sustainable development, as these projects provide new opportunities for industries and economic activities to setup in the area thereby resulting in greater local employment, ultimately leading to overall development. With respect to renewable energy there are great opportunities for India as in emerging markets renewable energy potential is attracting foreign investment, generating new jobs and 
Citation: Naik M, Singh A, Unnikrishnan S, Naik N, Nimkar I (2015) Review of Clean Development Mechanism and use of Bundled Projects in Small and Medium Scale Enterprises. Int J Waste Resour 5: 184. doi: 10.4172/2252-5211.1000184

Page 8 of 8

creating local supply chains. Government of India is also promoting use of renewable energy through its National Action Plan on Climate Change. The renewable energy goals require continues effort, strong implementation and improve utilization of capacity. Improvement in energy efficiency makes renewable energy source more affordable and attractive to finance source.

\section{Conclusion}

In recent years there has been increasing attention to the crucial issue of whether $\mathrm{CDM}$ has fulfilled its sustainable development objective. But the actual effects of CDM project activities on the host countries sustainable development at the national level are difficult to evaluate [30-33]. The global GHG emission reduction potential of such activities and empirical research is needed to identify and quantify actions that will yield the most emissions savings. Thus, the paper provides a probing insight into these small scale CDM project activities and their methodologies in order to achieve emission reduction. Small and medium scale industries occupy an important and strategic place in economic growth and equitable development in all the developing countries. Although CDM is devised to foster sustainable development, small scale CDM project activities which are known to be beneficial to the sustainable development of local communities, are often burdened with high costs and low returns. In order to leverage the development of small scale CDM projects, the UNFCCC introduced bundle concept. The concept is for the utilization of the CDM in simplified modalities and procedures for small-scale projects in order to secure carbon revenue for a community-scale. Small-scale project activities may be bundled up to the defined threshold level and eligible SSC project activities can be bundled to overcome transactions costs. By using bundle scheme, small projects can become cost effective and thus become sufficiently attractive with CER revenue.

\section{References}

1. Bharadwaj N, Parthan B, Coninck HC, Roos C, Van der Linden NH, et al. (2004) Realising the potential of small-scale CDM projects in India.

2. Bhardwaj B (2013) Future of Carbon Trading: A Business That Works For Global Environment. International Journal of Science, Environment \& Technology 2 : $115-121$

3. CDM rulebook (2013) What is bundling? Clean Development Mechanism rules, practice \& procedures

4. Central Electricity Authority (2013) CO2 Baseline Database for the Indian Power Sector User Guide Version 6.0, Ministry of Power, Government of India, New Delhi India.

5. Express hospitality (2013) Carbon credits: The Indian scenario.

6. Gonzales A (2001) Financing issues and options for small-scale industrial CDM projects in Asia.

7. Griffith-Jones S, Hedger M, Stokes $L$ (2009) The role of private investment in increasing climate friendly technologies in developing countries. Columbia University and Institute of Development Studies.

8. Haites E, Yamin F (2004) Special Feature on the Kyoto Protocol Overview of the Kyoto Mechanisms, International Review for Environmental Strategies 5 : 199-216.

9. Hinostroza M, Cheng CC, Zhu X, Fenhann J, Figueres C, et al. (2007) Potential and barriers for end-use energy efficiency under programmatic CDM. CD4CDM Working Paper 3, United Nation Environment programme, UNEP Riso Centre.

10. Huang Y, He J, Tarp F (2012) Is the Clean Development Mechanism Promoting Sustainable Development? Working paper, 2012/72, United Nation University.

11. Humbad A, Kumar S, Babu B (2009) Carbon credits for energy self-sufficiency in rural India- A case study. Energy science and research 22: 187-197.

12. IGES (2014) CDM project database.

13. International Renewable Energy Agency (2012) SUMMARY FOR POLICY MAKERS: Renewable Power Generation Costs.

14. Kumar HV, Kulkarni SV, Thukral K (2004) Bundled Small-Scale CDM Projects, UNEP Risoe Centre, Denmark.

15. Mariyappan J, Bharadwaj N, Conick H, Linden N (2005) A guide to bundled small-scale CDM projects.

16. Miah D, Shin MY, Koike M (2011) Forest to climate change mitigation. Clean development mechanism in Bangladesh, Springer Heidelberg Dordrecht London New York.

17. Ministry of New and Renewable Energy (MNRE) (2013) Physical Progress (Achievements). Government of India.

18. Najam A, Rahman A, Huq S, Sokona Y (2003) Integrating sustainable development into the Fourth Assessment Report of the Intergovernmental Panel on Climate Change. Climate Policy Volume 3: S9-S17.

19. Novikova A, Urge-Vorsatz D, Liang C (2006) The "Magic" of the Kyoto Mechanisms: Will It Work for Buildings?

20. Pan J (2009) Meeting Human Development Goals with Low Emissions. IDS Bulletin on Climate Change and Development 35: 90-97.

21. Schneider L (2007) Is the CDM Fulfilling its Environmental and Sustainable Development Objectives? An Evaluation of the CDM and Options fo Improvement. WWF Report.

22. Shishlov I, Bellassen V (2012) 10 Lessons from 10 Years of the CDM. Climate Report.

23. Shrestha RM, Abeygunawardana AMAK (2007) Small-scale CDM projects in a competitive electricity industry: How good is a simplified baseline methodology? Energy Policy 35: 3717-3728.

24. Sreekanth KJ, Sudarsan N, Jayaraj S (2012) Achieving Certified Emission Reduction in Rural Domestic Energy Sector Through Alternative Fue Replacement. International Journal of Renewable Energy Research 2: 38-43.

25. Subbarao S, Gadde B (2006) Analysis and Evaluation of CDM Potential of Biomethanation Sector in India. The 2nd Joint International Conference on "Sustainable Energy and Environment (SEE 2006)"F-024 (O) 21-23 November 2006, Bangkok, Thailand.

26. Sutter C (2001) Small-Scale CDM Projects: Opportunities and Obstacles.

27. The World Bank (2013) Mapping Carbon Pricing Initiatives report.

28. UNEP RISO CENRE (2013) Small Scale CDM \& Bundled Criteria, Advantages and Status.

29. UNFCCC (2013) Introduction to Clean Development Mechanism (CDM)

30. UNFCCC (2013) Annexure III, Bundles of small-scale CDM projects vs. largescale CDM projects.

31. UNFCCC (2013) CDM Methodology booklet. (6th edn) Information updated as of EB 79, United Nations

32. UNFCCC (2013) Clean development mechanism project design document (CDM-PDD).

33. World Bank (2013) Carbon Funds \& Facilities at the World Bank. 